\title{
Oxidized Low-Density Lipoprotein Predicts the Development of Renal Dysfunction in Atrial Fibrillation
}

\author{
Marija Polovinaa, c Ivana Petrovića ${ }^{a}$ oin Brković ${ }^{b, c} \quad$ Milika Ašanin a, c \\ Jelena Marinkovićc, d Miodrag Ostojićc \\ ${ }^{a}$ Cardiology Clinic and ${ }^{b}$ Nephrology Clinic, Clinical Center of Serbia, ${ }^{c}$ School of \\ Medicine, University of Belgrade, and d Department of Medical Statistics and Informatics, \\ Belgrade, Serbia
}

\section{Key Words}

Oxidative stress · Oxidized low-density lipoproteins · Atrial fibrillation · Chronic kidney

disease $\cdot$ Renal dysfunction - Glomerular filtration rate

\begin{abstract}
Background/Aim: To investigate the role of oxidative stress (OS) in the development of chronic kidney disease (CKD) in atrial fibrillation (AF). Methods: We compared OS burden, determined at study inclusion as plasma concentrations of oxidized low-density lipoprotein (oxLDL), between stable AF patients ( $n=256$, mean age: $62.8 \pm 9.3$ years; $60.9 \%$ males) with preserved renal function, defined as an estimated glomerular filtration rate (eGFR) $\geq 60 \mathrm{ml} /$ $\mathrm{min} / 1.73 \mathrm{~m}^{2}$, and a matched control group in sinus rhythm $(\mathrm{n}=138$, mean age: $61.5 \pm 11.2$ years; $60.9 \%$ males). During the prospective follow-up of AF patients, we investigated the association and prognostic validity of oxLDL for CKD development, diagnosed as a sustained decline in eGFR to $<60 \mathrm{ml} / \mathrm{min} / 1.73 \mathrm{~m}^{2}$. Results: AF patients had a higher mean oxLDL $(76.2$ $\pm 21.7 \mathrm{U} / \mathrm{l})$ compared to sinus rhythm controls $(61.6 \pm 13.1 \mathrm{U} / \mathrm{l} ; \mathrm{p}<0.001)$. AF presence independently predicted increased oxLDL levels in the study cohort $[\beta=14.7 ; 95 \%$ confidence interval (CI), 10.7-18.7; $p<0.001$ ]. Over a median 4-year follow-up, 19.9\% of AF patients developed CKD. Adjusting for all clinical covariates, oxLDL (per tertile) was associated with a hazard ratio of 2.17 for CKD occurrence (95\% CI, 1.40-3.35; $p<0.001)$. AF patients in the upper oxLDL tertile $(\geq 88.7 \mathrm{U} / \mathrm{l})$ had a 3.70 -fold $(95 \% \mathrm{CI}, 1.55-8.81)$ higher risk for CKD compared to the lower oxLDL tertile $(<67.0 \mathrm{U} / \mathrm{l})$ patients $(\mathrm{p}<0.001)$. oxLDL improved discriminative validity $(c-$ statistic increment: $0.041,95 \% \mathrm{CI}, 0.007-0.075, \mathrm{p}=0.017)$, and increased the net reclassifica-
\end{abstract}


tion and integrated discrimination for CKD risk by 12.4 and $6.0 \%$, respectively (both $p<0.001$ ). Conclusions: oxLDL is increased in AF patients compared to sinus rhythm controls. oxLDL has an independent association and an incremental predictive value that might complement clinical CKD risk assessment in AF patients following further research.

(C) 2016 S. Karger AG, Basel

\section{Background}

Atrial fibrillation (AF) is associated with an increased prevalence of chronic kidney disease (CKD) [1] usually defined as a sustained decrease in the glomerular filtration rate (GFR) below a threshold GFR of $<60 \mathrm{ml} / \mathrm{min} / 1.73 \mathrm{~m}^{2}$ [2]. The coexistence of AF and CKD accelerates CKD progression to end-stage renal disease [3], and confers a higher risk of adverse cardiovascular events and increased mortality in the affected patients [4, 5]. AF and CKD share predisposing conditions (e.g. hypertension, diabetes mellitus, and atherosclerosis) that could reflect common pathophysiological mechanisms, including low-grade inflammation, unbalanced production of reactive oxygen species (i.e. oxidative stress - OS), reninangiotensin system (RAS) activation, and endothelial dysfunction [6, 7]. Increased oxidative modification of plasma lipoproteins (e.g. oxidized low-density lipoproteins - oxLDL) has been documented in selected groups of AF [8] and CKD patients [9], and oxLDL has been implicated in the pathogenesis of cardiovascular and renal disorders by promoting endothelial dysfunction and vascular wall inflammation [10-12]. However, the role of oxLDL in the development of renal dysfunction in AF patients has not been characterized.

The aims of the present community-based, observational study were: (a) to compare plasma oxLDL levels determined at study inclusion between AF patients and a control group in sinus rhythm (SR), and (b) to assess whether baseline oxLDL levels are associated with CKD development in AF patients during the prospective follow-up. We also assessed whether oxLDL improved the prediction of CKD development beyond clinical and conventional laboratory predictors of renal dysfunction in AF patients.

\section{Methods}

Baseline Clinical Evaluation and Renal Function Assessment

Consecutive AF patients with electrocardiographically documented AF (paroxysmal, persistent and permanent) [13], treated as in- or outpatients at the Cardiology Clinic, Clinical Center of Serbia, between June 2010 and March 2011 were screened, and clinically stable patients with preserved renal function were included. A control group of comparable age, sex and comorbidities, with documented SR, no AF history, and preserved renal function was recruited at the same time among consecutive, stable in- and outpatients. The local Ethics Committee approved the study protocol, and informed consent was obtained from all participants.

Data on comorbidities and medical treatment were collected from medical records and/or diagnostic workup performed at inclusion, including: hypertension (blood pressure $\geq 140 / 90 \mathrm{~mm} \mathrm{Hg}$ or antihypertensive drug treatment); diabetes mellitus (fasting blood glucose $\geq 7.0 \mathrm{mmol} / \mathrm{l}$, and/or hemoglobin $\mathrm{A}_{1 \mathrm{c}}$ $\geq 6.5 \%$, or on treatment); echocardiographically documented aortic and/or mitral valve disease not requiring surgery; coronary artery disease (CAD) including prior myocardial infarction, percutaneous/surgical coronary revascularization or other angiographically documented CAD; history of heart failure (HF); history of stroke/transient ischemic attack (TIA)/systemic embolism; peripheral arterial disease (PAD), and chronic pulmonary disease. Baseline biochemistry and thyroid function analyses were performed, and transthoracic echocardiography was used to assess left ventricular ejection fraction, and the presence of a structural myocardial, and/or valvular disease. Baseline $\mathrm{CHA}_{2} \mathrm{DS}_{2}$-VASc stroke risk score was calculated in $\mathrm{AF}$ patients ( $\mathrm{C}$ = congestive $\mathrm{HF}, \mathrm{H}=$ hypertension, $\mathrm{A}_{2}=$ aged $\geq 75$ years - doubled, $\mathrm{D}=$ diabetes mellitus, $\mathrm{S}_{2}=$ stroke/TIA/ systemic embolism - doubled, $\mathrm{V}=$ vascular disease, $\mathrm{Sc}=$ females) [13]. 


\section{CardioRenal Medicine}

\begin{tabular}{l|l}
\hline Cardiorenal Med 2017;7:31-41 \\
\hline DOI: 10.1159/000449173 & $\begin{array}{l}\text { @ 2016 S. Karger AG, Basel } \\
\text { www.karger.com/crm }\end{array}$ \\
\hline
\end{tabular}

Polovina et al.: Oxidized Low-Density Lipoprotein Predicts the Development of Renal Dysfunction in Atrial Fibrillation

We excluded patients with conditions possibly influencing renal function and/or oxLDL, including: (1) hemodynamic instability (acute/decompensated chronic HF, current malignant ventricular arrhythmias), (2) acute AF causes (acute myocardial infarction, myopericarditis, thyrotoxicosis), (3) infections/inflammation, (4) valvular heart disease requiring surgery, (5) malignancy, and (6) trauma/surgery $\leq 3$ months ago.

In $\mathrm{AF}$ and $\mathrm{SR}$ patients screened for inclusion, serum creatinine was determined by a colorimetric Jaffe assay (Cobas 6000 Roche Diagnostics), and renal function was assessed by creatinine-based estimated GFR (eGFR), using the Chronic Kidney Disease Epidemiology Collaboration (CKD-EPI) equation considered to provide the most accurate GFR estimates [14]. Patients with baseline eGFR $\geq 60 \mathrm{ml} / \mathrm{min} / 1.73 \mathrm{~m}^{2}$ were considered to have preserved renal function and were included in the study [2,15]. Patients with either eGFR $<60 \mathrm{ml} / \mathrm{min} / 1.73 \mathrm{~m}^{2}$ or end-stage renal disease on renal replacement therapy were excluded.

\section{oxLDL Determination}

oxLDL was determined at inclusion. Fasting peripheral venous blood samples were taken in all participants, and following centrifugation $\left(2,200 \mathrm{rpm}, 20 \mathrm{~min}, 4^{\circ} \mathrm{C}\right)$, ethylenediaminetetraacetic acid plasma samples were stored in multiple aliquots at $-20^{\circ} \mathrm{C}$ for further analysis performed with a commercially available enzyme-linked immunosorbent (ELISA) assay for oxLDL (Mercodia, Uppsala, Sweden), with intraand interassay coefficients of variation of 6.8 and $7.1 \%$, respectively.

\section{Follow-Up and CKD Development in AF Patients}

AF patients were clinically followed and eGFR was reassessed at 3-month intervals. Study endpoint was CKD development, defined as a sustained eGFR decline to $<60 \mathrm{ml} / \mathrm{min} / 1.73 \mathrm{~m}^{2}$, documented on $\geq 2$ consecutive visits, $\geq 3$ months apart, in patients with baseline eGFR $\geq 60 \mathrm{ml} / \mathrm{min} / 1.73 \mathrm{~m}^{2}$. Lifestyle characteristics and medications potentially modifying renal function/OS (e.g. RAS inhibitors, statins) during the follow-up were in keeping with the contemporary guidelines $[2,13]$.

\section{Sample Size Calculation and Statistical Analysis}

For oxLDL comparison between AF and SR groups, a sample of at least 125 patients per group was calculated to detect a difference of $5 \mathrm{U} / \mathrm{l}$ in oxLDL means (standard deviation $=14 \mathrm{U} / \mathrm{l}$ ), with $\alpha=0.05$ and power $=0.8$, based on literature data [8,9]. Considering a prospective follow-up, a total sample of $255 \mathrm{AF}$ patients was calculated with a 95\% confidence level and 5\% precision, expecting CKD development in 20\% of AF patients, based on previous research [5], to allow for 5\% follow-up attrition.

Data are presented as means \pm standard deviations, medians (25th-75th interquartile ranges), or counts (n) with percentages (\%). The differences between variables were tested by the two-tailed Student $\mathrm{t}$ test, Mann-Whitney test, or $\chi^{2}$ test, as appropriate. The linear regression was used to analyze the association of AF with oxLDL, adjusted for age, sex, medications and baseline eGFR. Kaplan-Meier analysis was used to determine the cumulative incidence of CKD by oxLDL tertiles in AF patients and the differences in estimates were compared by the log-rank test. The association of oxLDL with CKD development in AF patients was analyzed by the Cox proportional hazard analysis, with oxLDL first entered as a continuous variable, and then categorized into tertiles (lower, middle and upper), chosen for providing the best data fitting with the study outcome. The association of baseline clinical and laboratory variables (listed in tables 1 and 2) with CKD development was assessed, and covariates significantly associated with CKD occurrence (table 3 ) were used to adjust the Cox model for CKD development with oxLDL. Possible interaction of statin treatment (potential OS modification) and oxLDL-related CKD risk was tested in the post hoc Cox analyses of treated versus nontreated patients. The interaction term (statin treatment $\times$ oxLDL per tertile) was included as a covariate in the analyses. In all analyses, the proportionality of hazards assumption was met and the time to CKD development or time to the last follow-up (for AF patients who did not develop CKD) was used. Hazard ratios (HR) and the $95 \%$ confidence intervals (CI) were calculated using the regression coefficients and their standard errors. For oxLDL tertiles, HR comparing the middle and upper tertiles with the lower tertile (reference) were reported. The ability to discriminate patients who would and would not develop CKD was first assessed by the area under the receiver operating characteristic (ROC) curve of the prediction model, with clinical covariates associated with CKD development (the $c$-statistic-1) and then with the prediction model including clinical covariates and oxLDL (the $c$-statistic-2) [16]. The incremental discriminative value of oxLDL was assessed by comparing the areas under the ROC curves ( $\Delta c$-statistic) before ( $c$-statistic- 1$)$ and after oxLDL inclusion into the model ( $c$-statistic-2) [16]. The Hosmer-Lemeshow goodness-of-fit test was used to assess the calibration of the model with oxLDL to match the estimated with the observed proportion of CKD development [17]. The net reclassification improvement (NRI) and integrated discrimination improvement (IDI) 
Polovina et al:: Oxidized Low-Density Lipoprotein Predicts the Development of Renal Dysfunction in Atrial Fibrillation

Table 1. Baseline demographic and clinical characteristics of AF patients and SR controls

\begin{tabular}{|c|c|c|c|}
\hline Baseline characteristics & $\begin{array}{l}\text { AF patients } \\
(\mathrm{n}=256)\end{array}$ & $\begin{array}{l}\text { SR controls } \\
(n=138)\end{array}$ & $\mathrm{p}$ value \\
\hline Age, years & $\begin{array}{l}62.8 \pm 9.3 \\
\text { (range: } 29-85 \text { ) }\end{array}$ & $\begin{array}{l}61.5 \pm 11.2 \\
\text { (range: } 27-83 \text { ) }\end{array}$ & 0.210 \\
\hline Sex, males & $156(60.9)$ & $84(60.9)$ & 0.989 \\
\hline \multicolumn{4}{|l|}{ AF type } \\
\hline Paroxysmal AF & $124(48.4)$ & - & - \\
\hline Persistent AF & $63(24.6)$ & - & - \\
\hline Permanent AF & $69(27.0)$ & - & - \\
\hline Body mass index & $27.0 \pm 3.2$ & $26.4 \pm 3.1$ & 0.081 \\
\hline \multicolumn{4}{|l|}{ Comorbidities and risk factors } \\
\hline Hypertension & $234(91.4)$ & $127(92.0)$ & 0.831 \\
\hline Diabetes mellitus & $40(15.6)$ & $17(12.3)$ & 0.373 \\
\hline Coronary artery disease & $37(14.5)$ & $18(13.0)$ & 0.700 \\
\hline Mild/moderate mitral valve disease $\mathrm{a}^{\mathrm{a}}$ & $71(27.7)$ & $42(30.4)$ & 0.572 \\
\hline Mild/moderate aortic valve disease ${ }^{b}$ & $2(0.8)$ & $2(1.4)$ & 0.528 \\
\hline Prior heart failure & $7(2.7)$ & $5(3.6)$ & 0.624 \\
\hline Current left ventricular ejection fraction, $\%$ & $59.2 \pm 7.5$ & $58.3 \pm 4.6$ & 0.570 \\
\hline Prior stroke/TIA/systemic embolism & $24(9.4)$ & $4(2.9)$ & 0.017 \\
\hline Peripheral arterial disease & $8(3.1)$ & $5(3.6)$ & 0.792 \\
\hline Chronic pulmonary disease & $5(2.0)$ & $6(4.4)$ & 0.161 \\
\hline $\mathrm{CHA}_{2} \mathrm{DS}_{2}$-VASc score & $2.4 \pm 1.3$ & - & - \\
\hline Current smokers & $28(10.9)$ & $14(10.1)$ & 0.875 \\
\hline Alcohol consumption, moderate & $94(36.7)$ & $47(34.0)$ & 0.176 \\
\hline \multicolumn{4}{|l|}{ Medical therapy } \\
\hline$\beta$-Blockers & $134(52.3)$ & $68(49.3)$ & 0.736 \\
\hline Non-dihydropyridine $\mathrm{Ca}^{2+}$ antagonists & $9(11.3)$ & $0(0.0)$ & $<0.001$ \\
\hline Digoxin & $21(8.2)$ & $0(0.0)$ & $<0.001$ \\
\hline Amiodarone & $102(39.8)$ & $3(2.2)$ & $<0.001$ \\
\hline ACE inhibitors $/ \mathrm{AT}_{1}$ receptor blockers & $202(78.9)$ & $114(82.6)$ & 0.739 \\
\hline Dihydropyridine $\mathrm{Ca}^{2+}$ antagonists & $76(29.7)$ & $36(26.1)$ & 0.450 \\
\hline Loop diuretics & $37(14.5)$ & $17(12.3)$ & 0.651 \\
\hline Thiazide diuretics & $113(44.1)$ & $66(47.8)$ & 0.765 \\
\hline Statins & $119(46.5)$ & $68(49.2)$ & 0.154 \\
\hline Aspirin & $53(20.7)$ & $26(18.8)$ & 0.312 \\
\hline Oral anticoagulants & $214(83.6)$ & $0(0.0)$ & $<0.001$ \\
\hline
\end{tabular}

were used to assess whether oxLDL improved reclassification of patients' predicted probability for developing CKD compared to classification based on clinical predictors [18]. A p value of $<0.05$ denoted statistical significance. Analyses were performed using statistical packages: SPSS v22 (SPSS, Inc. Chicago, Ill., USA), MedCalc v12.7.0.0, and Stata/MP-14 (StataCorp LP).

\section{Results}

\section{Baseline Characteristics of the Study Population}

Of 314 consecutive, stable AF patients screened for the study, preserved renal function (eGFR $\geq 60 \mathrm{ml} / \mathrm{min} / 1.73 \mathrm{~m}^{2}$ ) was documented in 256 patients $(81.5 \%$ ), and those patients comprised the AF group (mean age: $62.8 \pm 9.3$ years; $60.9 \%$ males). The control group included 
Table 2. Baseline laboratory characteristics

\begin{tabular}{lccr}
\hline Laboratory characteristics & $\begin{array}{l}\text { AF patients } \\
(\mathrm{n}=256)\end{array}$ & $\begin{array}{l}\text { SR controls } \\
(\mathrm{n}=138)\end{array}$ & p value \\
\hline $\begin{array}{l}\text { Renal function } \\
\quad \text { Serum urea, } \mathrm{mmol} / \mathrm{l}\end{array}$ & $5.9 \pm 1.5$ & $5.3 \pm 1.2$ & 0.019 \\
$\quad$ Serum creatinine, $\mu \mathrm{mol} / \mathrm{l}$ & $80.3 \pm 14.0$ & $71.2 \pm 10.0$ & $<0.001$ \\
eGFR, $\mathrm{ml} / \mathrm{min} / 1.73 \mathrm{~m}^{2}$ & $81.2 \pm 12.6$ & $89.2 \pm 10.3$ & $<0.001$ \\
eGFR $\geq 90 \mathrm{ml} / \mathrm{min} / 1.73 \mathrm{~m}^{2}$ & $70(27.3)$ & $55(39.9)$ & 0.011 \\
eGRF $\geq 60$ to $<90 \mathrm{ml} / \mathrm{min} / 1.73 \mathrm{~m}^{2}$ & $186(72.7)$ & $83(60.1)$ & 0.011 \\
Plasma lipids & $5.4 \pm 1.2$ & $5.5 \pm 1.1$ & 0.112 \\
Total cholesterol, $\mathrm{mmol} / \mathrm{l}$ & $1.2 \pm 0.4$ & $1.2 \pm 0.8$ & 0.134 \\
HDL cholesterol, $\mathrm{mmol} / \mathrm{l}$ & $3.6 \pm 1.1$ & $3.6 \pm 0.9$ & 0.211 \\
LDL cholesterol, $\mathrm{mmol} / \mathrm{l}$ & $1.7 \pm 0.8$ & $1.6 \pm 0.9$ & 0.088 \\
Triglycerides, mmol/l & $76.2 \pm 21.7$ & $61.6 \pm 13.1$ & $<0.001$ \\
$\quad$ oxLDL, U/l & & \\
\hline
\end{tabular}

Table 3. Clinical and laboratory characteristics associated with CKD development in AF patients

\begin{tabular}{lcccr}
\hline Patient characteristics & $\begin{array}{l}\text { AF patients } \\
\text { without CKD } \\
\text { development } \\
\text { (n=205) }\end{array}$ & $\begin{array}{l}\text { AF patients } \\
\text { with CKD } \\
\text { development } \\
(\mathrm{n}=51)\end{array}$ & HR (95\% CI) & p value \\
\hline Age, years & $61.4 \pm 9.4$ & $68.6 \pm 6.4$ & $1.09(1.05-1.13)$ & $<0.001$ \\
Permanent AF & $47(22.9)$ & $22(43.1)$ & $2.36(1.35-4.11)$ & 0.002 \\
Diabetes mellitus & $25(12.2)$ & $15(29.4)$ & $2.51(1.37-4.59)$ & 0.003 \\
Coronary artery disease & $24(11.7)$ & $13(25.5)$ & $2.52(1.34-4.74)$ & 0.004 \\
Mitral valve disease & $47(22.9)$ & $24(47.1)$ & $2.77(1.59-4.81)$ & $<0.001$ \\
Prior stroke/TIA/systemic embolism & $13(6.3)$ & $11(21.6)$ & $2.78(1.43-5.43)$ & 0.003 \\
PAD & $4(2.0)$ & $4(7.8)$ & $3.79(1.36-8.55)$ & 0.003 \\
CHA ${ }_{2}$ DS ${ }_{2}$-VASc score & $2.1 \pm 1.1$ & $3.3 \pm 1.5$ & $1.74(1.47-2.90)$ & $<0.001$ \\
Baseline eGFR, ml/min/1.73 m ${ }^{2}$ & $83.7 \pm 12.3$ & $71.3 \pm 8.6$ & $6.73(2.78-10.71)^{\mathrm{a}}$ & $<0.001$ \\
High-density lipoprotein cholesterol, & $1.2 \pm 0.3$ & $0.8 \pm 0.5$ & $1.76(1.06-2.92)^{\mathrm{b}}$ & 0.028 \\
$\quad$ mmol/l $^{2}$ & & & & \\
\hline
\end{tabular}

Variables are presented as: mean \pm standard deviation or $\mathrm{n}(\%)$.

${ }^{a} \mathrm{HR}$ is expressed per $30 \mathrm{ml} / \mathrm{min} / 1.73 \mathrm{~m}^{2} \mathrm{GFR}$ decrease. ${ }^{\mathrm{b}} \mathrm{HR}$ is expressed per quartile decrease in Highdensity lipoprotein cholesterol concentration.

138 stable patients in SR, with preserved renal function (mean age: $61.5 \pm 11.2$ years; $60.9 \%$ males). Baseline characteristics of both groups are presented in table 1 . The two groups were comparable regarding age, sex and comorbidities, except for the lower frequency of prior stroke among SR controls. Expectedly, differences regarding the use of antiarrhythmic/anticoagulant drugs were observed between the groups (table 1).

Baseline laboratory characteristics are presented in table 2. Compared to the controls, AF patients had a higher mean serum creatinine, a lower mean eGFR, a lower proportion of subjects with eGFR $\geq 90 \mathrm{ml} / \mathrm{min} / 1.73 \mathrm{~m}^{2}$ and a higher proportion of patients with eGRF $\geq 60$ to $<90 \mathrm{ml} / \mathrm{min} / 1.73 \mathrm{~m}^{2}$ (all $\mathrm{p}<0.05$ ) (table 2). No differences in plasma lipids were observed. The mean oxLDL of AF patients $(76.2 \pm 21.7 \mathrm{U} / \mathrm{l})$ was higher compared to SR controls $(61.6 \pm$ 


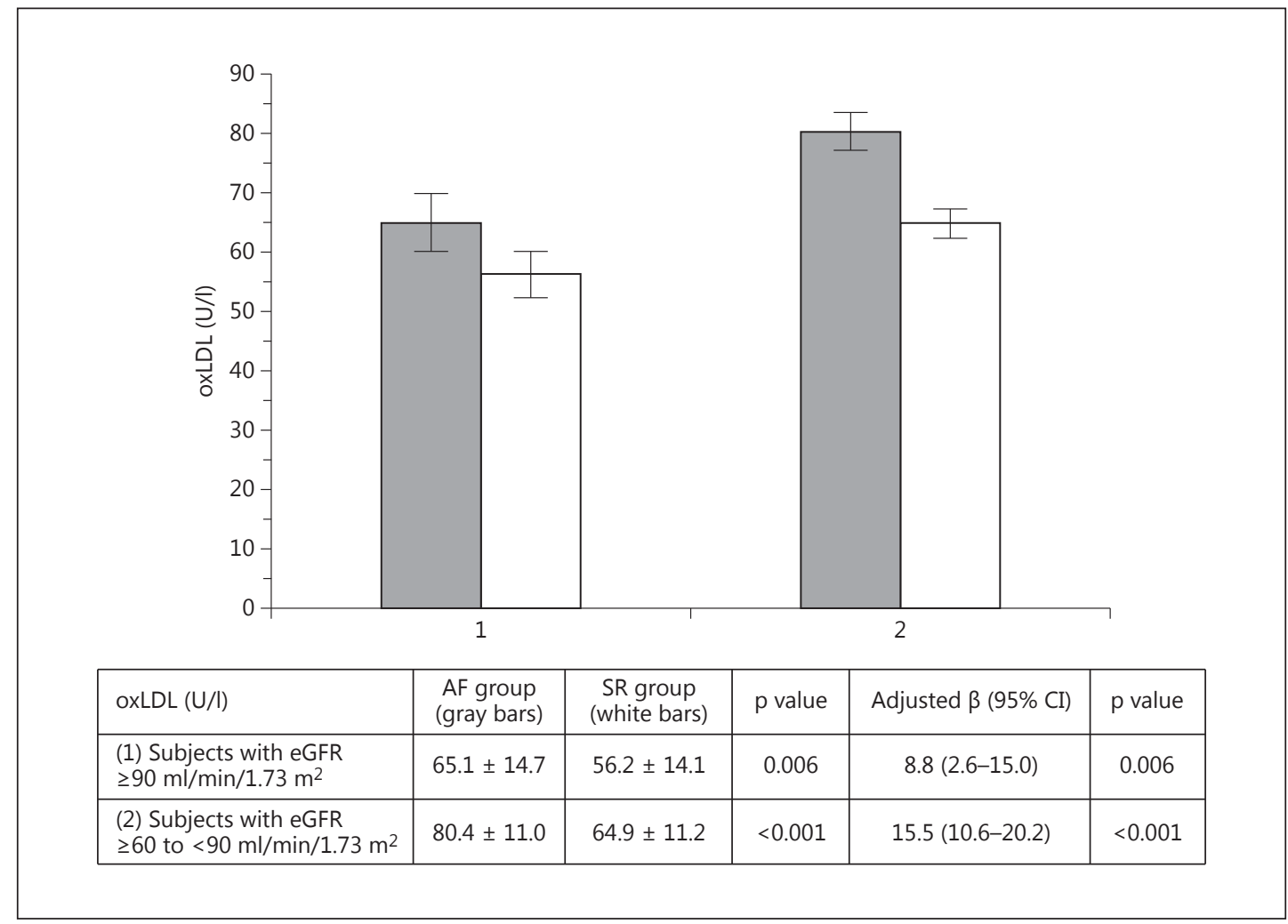

Fig. 1. oxLDL levels according to baseline eGFR in AF patients (gray bars) and SR controls (white bars).

$13.1 \mathrm{U} / \mathrm{l} ; \mathrm{p}<0.001$ ) (table 2). The comparison of oxLDL between AF and SR patients divided into subgroups with 'higher' (eGFR $\geq 90 \mathrm{ml} / \mathrm{min} / 1.73 \mathrm{~m}^{2}$ ) and 'lower' (eGRF $\geq 60$ to $<90 \mathrm{ml} /$ $\mathrm{min} / 1.73 \mathrm{~m}^{2}$ ) baseline renal function also demonstrated higher oxLDL in AF subjects (both $\mathrm{p}<0.05$ ) (fig. 1). In the regression model adjusted for age, sex, medications and eGFR, AF presence predicted higher oxLDL levels in the entire study cohort $(\beta=14.7$; $95 \% \mathrm{CI}, 10.7-$ 18.7; $\mathrm{p}<0.001$ ), as well as in the subgroups with 'higher' and 'lower' baseline renal function (fig. 1).

\section{CKD Development in AF Patients - Clinical and Conventional Laboratory Predictors}

After a median of a 48-month follow-up (range: 4-66 months), CKD was established in $51(19.9 \%)$ patients. Of those patients, eGFR decreased to $31-60 \mathrm{ml} / \mathrm{min} / 1.73 \mathrm{~m}^{2}, 15-30 \mathrm{ml} /$ $\mathrm{min} / 1.73 \mathrm{~m}^{2}$ and $<15 \mathrm{ml} / \mathrm{min} / 1.73 \mathrm{~m}^{2}$ in 43,6 and 2 patients, respectively (1 patient commenced dialysis). Clinical and laboratory predictors of CKD development are presented in table 3 .

\section{Association of oxLDL with CKD Development in AF Patients}

The lower, middle and upper oxLDL tertiles in AF patients were defined by the following cutoffs: $<67.0 \mathrm{U} / \mathrm{l}, \geq 67.0$ to $<88.7 \mathrm{U} / \mathrm{l}$, and $\geq 88.7 \mathrm{U} / \mathrm{l}$, respectively. There was a graded increase in the cumulative incidence of CKD associated with increasing oxLDL tertiles (log-rank $\mathrm{p}<$ 0.001 ) (fig. 2). oxLDL (per tertile) conferred an unadjusted HR of 2.24 (95\% CI, 1.55-3.23) for CKD development, while the risk of CKD associated with the middle $(\geq 67.0$ to $<88.7 \mathrm{U} / \mathrm{l}$ ) and upper ( $\geq 88.7 \mathrm{U} / \mathrm{l}$ ) oxLDL tertiles increased 3.10-fold (95\% CI, 1.29-7.84) and 5.64-fold (95\% CI, 2.46-12.98), respectively, relative to the lower oxLDL tertile $(<67.0 \mathrm{U} / \mathrm{l}$; all $\mathrm{p}<0.05)$ 
Polovina et al:: Oxidized Low-Density Lipoprotein Predicts the Development of Renal Dysfunction in Atrial Fibrillation

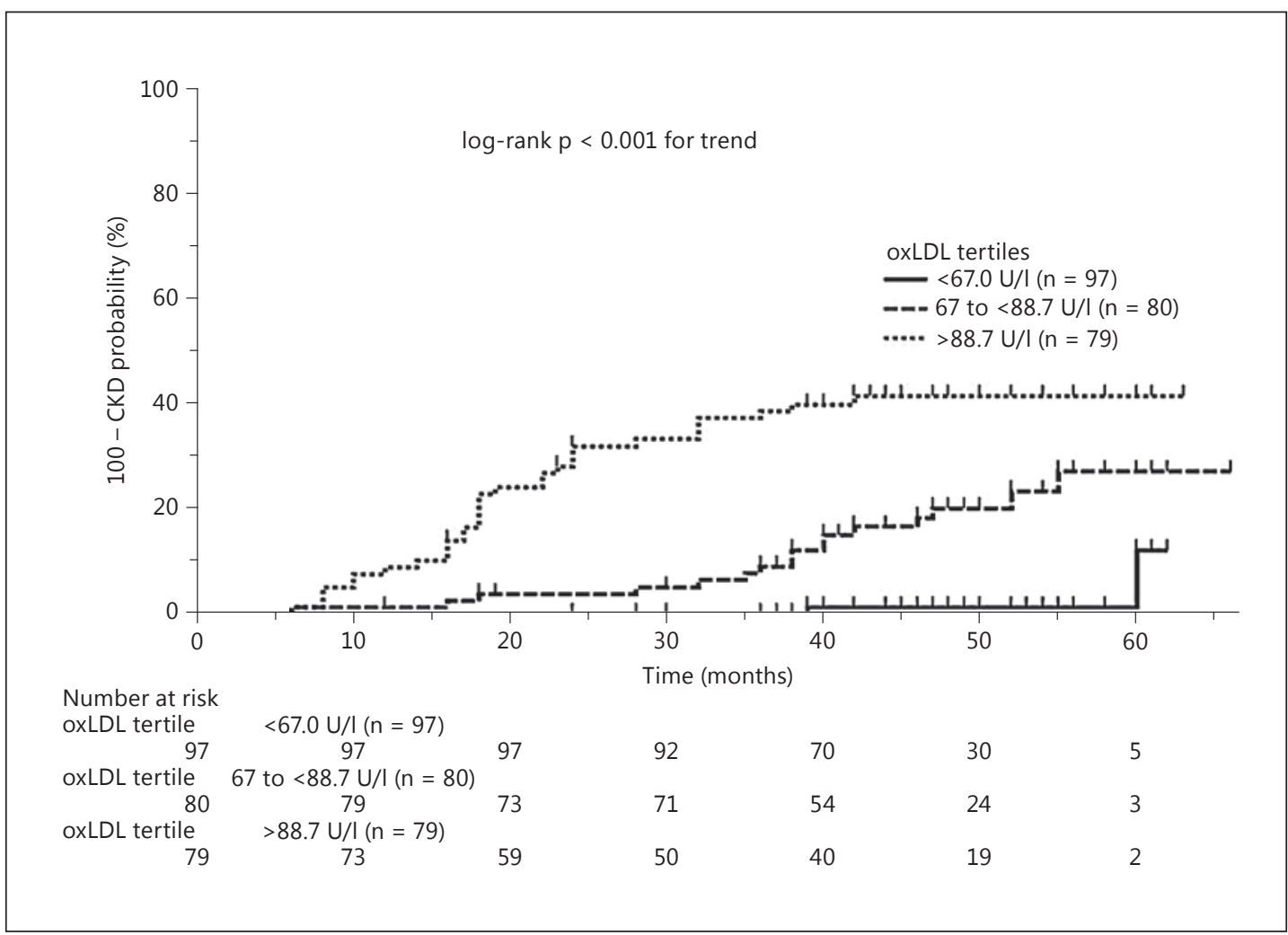

Fig. 2. Cumulative incidence of CKD development according to baseline oxLDL tertiles.

Table 4. Association of oxLDL with CKD development in AF patients

\begin{tabular}{|c|c|c|c|c|}
\hline oxLDL association with CKD & $\begin{array}{l}\text { Unadjusted HR } \\
(95 \% \mathrm{CI})\end{array}$ & $\mathrm{p}$ value & $\begin{array}{l}\text { Adjusted }^{\mathrm{a}} \mathrm{HR} \\
(95 \% \mathrm{CI})\end{array}$ & $\mathrm{p}$ value \\
\hline oxLDL (U/l) & $1.04(1.02-1.05)$ & $<0.001$ & $1.03(1.01-1.05)$ & $<0.001$ \\
\hline oxLDL (per tertile) & $2.24(1.55-3.23)$ & $<0.001$ & $2.17(1.40-3.35)$ & $<0.001$ \\
\hline oxLDL (lower tertile, <67.0 U/l) & 1 & - & 1 & - \\
\hline \multicolumn{5}{|l|}{ oxLDL (middle tertile, } \\
\hline$\geq 67.0$ to $<88.7 \mathrm{U} / \mathrm{l})$ & $3.10(1.29-7.84)$ & 0.012 & $2.10(0.86-5.14)$ & 0.103 \\
\hline oxLDL (upper tertile, $\geq 88.7 \mathrm{U} / \mathrm{l}$ ) & $5.64(2.46-12.98)$ & $<0.001$ & $3.70(1.55-8.81)$ & $<0.001$ \\
\hline
\end{tabular}

a Adjusted for clinical covariates listed in table 3.

(table 4). Following adjustments for clinical covariates from table 3, oxLDL (per tertile) was associated with an HR of 2.17 for subsequent CKD (95\% CI, 1.40-3.35, p < 0.001), while patients in the upper oxLDL tertile ( $\geq 88.7 \mathrm{U} / \mathrm{l}$ ) had a 3.70 -fold ( $95 \% \mathrm{CI} 1.55-8.81$ ) higher risk for CKD development compared to patients in the lower tertile $(<67.0 \mathrm{U} / \mathrm{l}, \mathrm{p}<0.001)$ (table 4 ). The association of oxLDL (per tertile) with CKD risk was consistent, irrespective of a potential OS-modifying effect of statin treatment [HRs, treated vs. nontreated patients, 2.19 (95\% CI, $1.43-3.29$ ) vs. 2.30 ( $95 \% \mathrm{CI}, 1.49-3.21$ ), respectively; $\mathrm{p}=0.465$ for interaction].

Inclusion of oxLDL into the model based on clinical CKD predictors produced a significant $c$-statistic increment of 0.041 ( $95 \% \mathrm{CI}, 0.007-0.075, \mathrm{p}=0.017$ ), as presented in figure 3 . The prediction model with oxLDL was well calibrated $\left(\chi^{2}=6.970, p=0.540\right.$, nonsignificant $p$ value 
Polovina et al:: Oxidized Low-Density Lipoprotein Predicts the Development of Renal Dysfunction in Atrial Fibrillation

Fig. 3. Comparison of the areas under the ROC curves $(\Delta c$ statistic) for the risk of CKD development with (B) and without oxLDL (A).

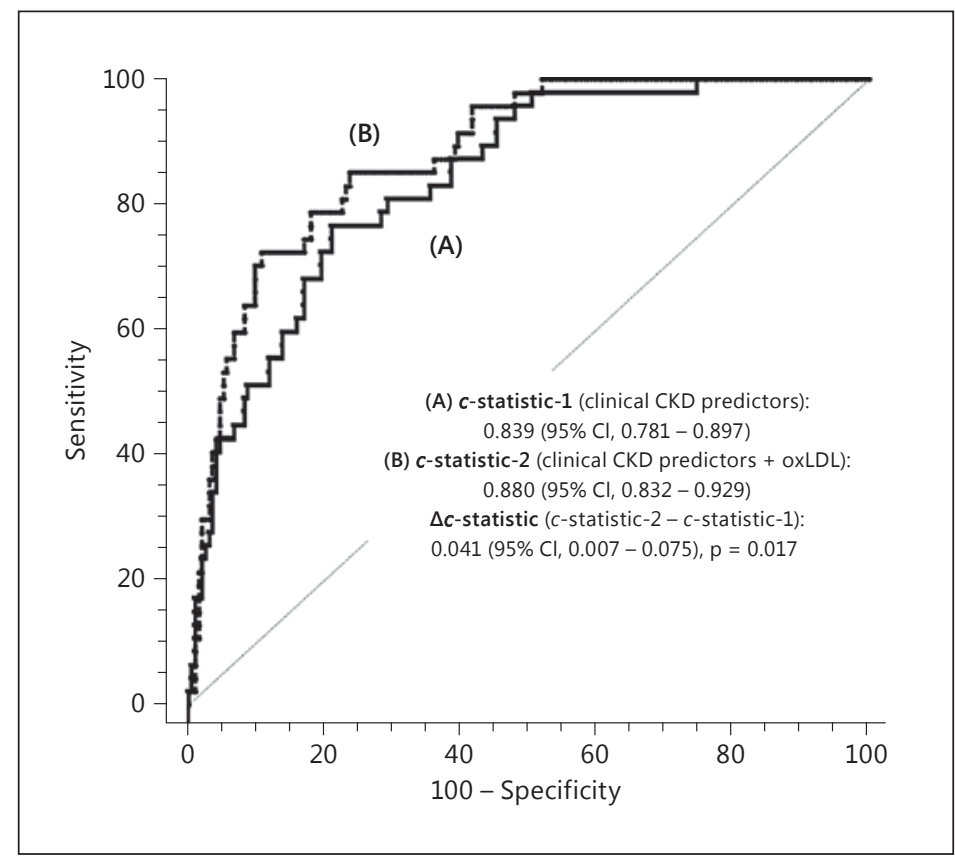

denotes adequate calibration), and provided an improved reclassification of the predicted probability for CKD development, as reflected by an increase in NRI and IDI of 12.4 and $6.0 \%$, respectively (both $\mathrm{p}<0.001$ ), compared to clinical CKD predictors.

\section{Discussion}

The main findings of the present community-based, observational study indicate that OS, as determined by plasma oxLDL, was higher in stable AF patients with preserved renal function compared to SR controls. AF presence was associated with increased oxLDL independent of clinical characteristics and eGFR. Importantly, it was documented for the first time that baseline oxLDL was associated with an increased risk of CKD development during the prospective 4-year median follow-up of AF patients. Inclusion of oxLDL improved discrimination and reclassification properties of the model based on clinical and laboratory CKD predictors, indicating that oxLDL might add to an improved recognition of CKD risk in AF patients.

oxLDL has been established as a marker of in vivo lipid peroxidation reflecting increased OS under pathological conditions [19]. To date, several case-control studies have reported increased oxLDL in the general CKD population $[9,20]$, as well as in AF patients [21], and recently, oxLDL has been linked to the risk of developing cardiovascular disease in AF patients without associated comorbidities [22]. Our findings extend those observations to the interplay of AF, OS and renal dysfunction. Among subjects with preserved renal function, we found lower mean eGFR in AF patients compared to SR controls, which is in line with the reported higher prevalence of AF-associated renal impairment [1]. Nevertheless, when subjects with similar eGFR were compared, AF patients demonstrated higher oxLDL compared to SR controls. Notably, AF presence was an independent predictor of increased oxLDL in the entire study cohort, as well as in subjects with 'higher' (eGFR $\geq 90 \mathrm{ml} / \mathrm{min} / 1.73 \mathrm{~m}^{2}$ ) and with 'lower' (eGFR $\geq 60$ to $<90 \mathrm{ml} / \mathrm{min} / 1.73 \mathrm{~m}^{2}$ ) baseline renal function, strengthening the notion for increased OS burden in AF. 
The present study provides evidence for an association of oxLDL with a risk of CKD in patients with AF. In our cohort, $19.9 \%$ of initially stable AF patients developed CKD over a median of 4 years, confirming earlier observations of a relatively high incidence of CKD in AF (e.g. $21 \%$ over the 2 years) [5]. In the present study, oxLDL was strongly associated with CKD development, independent of clinical covariates, and added prognostic information to clinical predictors, as demonstrated by an improvement in discrimination and reclassification indices. These observations suggest that oxLDL could complement clinical risk factors in assessing patients' risk of CKD. Whether oxLDL acts as an initiator, a contributor or an associate of renal dysfunctions is still obscure. oxLDL has been primarily investigated in the context of atherosclerosis [10,23], while mechanisms involved in kidney impairment have remained elusive. oxLDL could advance renal dysfunction by promoting vascular damage, and in particular (microvascular) endothelial dysfunction [11], which in turn may intensify OS, inflammation and RAS activation that perpetuate disease progression. Increased OS could also explain a bidirectional relationship of AF and CKD, as well as an increased burden of cardiovascular morbidity and mortality observed in patients affected by both conditions [4].

Clinical risk factors specific for CKD development in AF have not been widely addressed. Similar to previous findings $[5,24]$, the risk of CKD in AF patients in the present study increased with advancing age and declining baseline eGFR, as well as the presences of diabetes mellitus, cardiovascular comorbidities (i.e. CAD, PAD, mitral valve disease), and prior thromboembolism. Interestingly, permanent AF also increased the risk of renal dysfunction, possibly reflecting chronic adverse hemodynamic influences, or increased thromboembolic burden. Similar to observations from the general population [25], our results demonstrated that low high-density lipoprotein cholesterol, but not LDL cholesterol, predicted an increased risk of renal dysfunction, implying that deleterious effects of dyslipidemia do not provide a full explanation for the association of oxLDL with CKD [12]. Additionally, oxLDL-related CKD risk was consistent regardless of the treatment with statins, despite their lipid-lowering and (potential) OS-modifying properties [2]. However, this finding might be biased by inadequate statistical power, or treatment changes over the time course of the study. Furthermore, we have observed an association of CKD development with a higher baseline $\mathrm{CHA}_{2} \mathrm{DS}_{2}$-VASc score, likely attributable to a less favorable risk factor profile of patients with increased $\mathrm{CHA}_{2} \mathrm{DS}_{2}$-VASc scores, and vice versa, higher $\mathrm{CHA}_{2} \mathrm{DS}_{2}$-VASc scores might have produced a higher burden of (silent) thromboembolic damage to the kidneys, spurring the progression of renal dysfunction during the follow-up.

\section{Study Limitations}

Several limitations deserve consideration regarding the present study. Firstly, a singlecenter observational design and inclusion of a relatively small number of stable patients with preserved eGFR limit generalizability of our findings. In particular, we have not investigated whether oxLDL predicted renal function deterioration in subjects with established CKD. Also, a potential association of oxLDL with CKD development in SR subjects was beyond the scope of the present study. Although creatinine-based eGFR is a well-established marker of the overall renal function [15], we have not evaluated other indices of renal injury/dysfunction (i.e. albuminuria/proteinuria, cystatin C, etc.) and their association with oxLDL and CKD. Also, one-off oxLDL measurement at study inclusion prevented time-dependent Cox analysis of the study outcome. Despite adjustments for a number of covariates, the observed associations could have resulted from unappreciated clinical/laboratory confounders. Finally, other markers of OS have not been assessed to substantiate the association of OS with renal impairment. Therefore, a confirmation from a larger study in the general AF population is necessary to conclude the role of oxLDL in AF and CKD. 


\section{CardioRenal Medicine}

Polovina et al.: Oxidized Low-Density Lipoprotein Predicts the Development of Renal Dysfunction in Atrial Fibrillation

\section{Conclusions}

Our findings indicate that $\mathrm{AF}$ is associated with higher OS burden compared to SR. oxLDL has independent and incremental predictive implications for CKD development, with a potential to complement clinical risk assessment for CKD in AF patients. From the clinical perspective, defining individual risk for CKD could guide preventive lifestyle/pharmacological interventions and improve management in challenging clinical situations. Further research is warranted to investigate pathophysiological correlates of OS in AF and renal dysfunction, and to validate clinical implications of oxLDL for CKD risk stratification in AF.

\section{Acknowledgement}

The research was supported by the Republic of Serbia, Ministry of Science, Research Project No. 41022. The authors have no personal financial disclosures regarding the research.

\section{Statement of Ethics}

The Ethics Committees of the Clinical Center of Serbia and the School of Medicine, Belgrade University approved the study protocol, and the subjects provided written informed consent.

\section{Disclosure Statement}

The authors declare no conflicts of interest.

\section{References}

1 Kooiman J, van de Peppel WR, van der Meer FJ, Huisman MV: Incidence of chronic kidney disease in patients with atrial fibrillation and its relevance for prescribing new oral antithrombotic drugs. J Thromb Haemost 2011;9:1652-1653.

2 Improving Global Outcomes (KDIGO) CKD Work Group: KDIGO 2012 clinical practice guideline for the evaluation and management of chronic kidney disease. Kidney Inter Suppl 2013;3:1-150.

3 Bansal N, Fan D, Hsu CY, Ordonez JD, Marcus GM, Go AS: Incident atrial fibrillation and risk of end-stage renal disease in adults with chronic kidney disease. Circulation 2013;127:569-574.

4 Olesen JB, Lip GY, Kamper AL, Hommel K, Kober L, Lane DA, et al: Stroke and bleeding in atrial fibrillation with chronic kidney disease. N Engl J Med 2012;367:625-635.

5 Roldan V, Marin F, Fernandez H, Manzano-Fernandez S, Gallego P, Valdes M, et al: Renal impairment in a 'reallife' cohort of anticoagulated patients with atrial fibrillation (implications for thromboembolism and bleeding). Am J Cardiol 2013;111:1159-1164.

6 Schiffrin EL, Lipman ML, Mann JF: Chronic kidney disease: effects on the cardiovascular system. Circulation 2007;116:85-97.

7 Polovina MM, Lip GY, Potpara TS: Endothelial (dys)function in lone atrial fibrillation. Curr Pharm Des 2015; 21:622-645.

8 Tousoulis D, Zisimos K, Antoniades C, Stefanadi E, Siasos G, Tsioufis C, et al: Oxidative stress and inflammatory process in patients with atrial fibrillation: the role of left atrium distension. Int J Cardiol 2009;136:258-262.

9 Johnson-Davis KL, Fernelius C, Eliason NB, Wilson A, Beddhu S, Roberts WL: Blood enzymes and oxidative stress in chronic kidney disease: a cross sectional study. Ann Clin Lab Sci 2011;41:331-339.

10 Meisinger C, Baumert J, Khuseyinova N, Loewel H, Koenig W: Plasma oxidized low-density lipoprotein, a strong predictor for acute coronary heart disease events in apparently healthy, middle-aged men from the general population. Circulation 2005;112:651-657.

11 Pirillo A, Norata GD, Catapano AL: LOX-1, OxLDL, and atherosclerosis. Mediators Inflamm 2013;2013:152786.

12 Omran J, Al-Dadah A, Dellsperger KC: Dyslipidemia in patients with chronic and end-stage kidney disease. Cardiorenal Med 2013;3:165-177.

13 Camm AJ, Kirchhof P, Lip GY, Schotten U, Savelieva I, Ernst S, et al: Guidelines for the management of atrial fibrillation: the Task Force for the Management of Atrial Fibrillation of the European Society of Cardiology (ESC). Europace 2010;12:1360-1420. 
Polovina et al.: Oxidized Low-Density Lipoprotein Predicts the Development of Renal Dysfunction in Atrial Fibrillation

14 Levey AS, Stevens LA, Schmid CH, Zhang YL, Castro AF 3rd, Feldman HI, et al: A new equation to estimate glomerular filtration rate. Ann Intern Med 2009;150:604-612.

15 Boriani G, Savelieva I, Dan GA, Deharo JC, Ferro C, Israel CW, et al: Chronic kidney disease in patients with cardiac rhythm disturbances or implantable electrical devices: clinical significance and implications for decision making-a position paper of the European Heart Rhythm Association endorsed by the Heart Rhythm Society and the Asia Pacific Heart Rhythm Society. Europace 2015;17:1169-1196.

16 DeLong ER, DeLong DM, Clarke-Pearson DL: Comparing the areas under two or more correlated receiver operating characteristic curves: a nonparametric approach. Biometrics 1988;44:837-845.

17 Hosmer DW, Hosmer T, Le Cessie S, Lemeshow S: A comparison of goodness-of-fit tests for the logistic regression model. Stat Med 1997;16:965-980.

18 Pencina MJ, D’Agostino RB Sr, D’Agostino RB Jr, Vasan RS: Evaluating the added predictive ability of a new marker: from area under the ROC curve to reclassification and beyond. Stat Med 2008;27:157-172; discussion 207-212.

19 Itabe H, Yamamoto H, Imanaka T, Shimamura K, Uchiyama H, Kimura J, Sanaka T, Hata Y, Takano T: Sensitive detection of oxidatively modified low density lipoprotein using a monoclonal antibody. J Lipid Res 1996;37: 45-53.

20 Soltani A, Argani H, Soleimani F, Rahimipour H, Akbarzadeh-Baghban A, Azizi T, et al: Evaluation of serum oxidized low-density lipoprotein in renal transplant recipients and hemodialysis patients and relation with involved variables. Exp Clin Transplant 2015;13:524-528.

21 Idriss NK, Blann AD, Sayed DM, Gaber MA, Hassen HA, Kishk YT: Circulating endothelial cells and platelet microparticles in mitral valve disease with and without atrial fibrillation. Angiology 2015;66:631-637.

22 Polovina MM, Ostojic MC, Potpara TS: Relation of biomarkers of inflammation and oxidative stress with hypertension occurrence in lone atrial fibrillation. Mediators Inflamm 2015;2015:653026.

23 Li D, Liu L, Chen H, Sawamura T, Ranganathan S, Mehta JL: LOX-1 mediates oxidized low-density lipoproteininduced expression of matrix metalloproteinases in human coronary artery endothelial cells. Circulation 2003;107:612-617.

24 Bohm M, Ezekowitz MD, Connolly SJ, Eikelboom JW, Hohnloser SH, Reilly PA, et al: Changes in renal function in patients with atrial fibrillation: an analysis from the RE-LY Trial. J Am Coll Cardiol 2015;65:2481-2493.

25 Muntner P, Coresh J, Smith JC, Eckfeldt J, Klag MJ: Plasma lipids and risk of developing renal dysfunction: the atherosclerosis risk in communities study. Kidney Int 2000;58:293-301. 